\title{
Probabilistic quantum phase-space simulation of Bell violations and their dynamical evolution
}

\author{
L. Rosales-Zárate ${ }^{1}$, B. Opanchuk ${ }^{1}$, P. D. Drummond ${ }^{1}$, and M. D. Reid ${ }^{1}$ \\ ${ }^{1}$ Centre for Quantum and Optical Science, Swinburne University of Technology, Melbourne 3122, Australia
}

\begin{abstract}
Quantum simulations of Bell inequality violations are numerically obtained using probabilistic phase space methods, namely the positive P-representation. In this approach the moments of quantum observables are evaluated as moments of variables that have values outside the normal eigenvalue range. There is thus a parallel with quantum weak measurements and weak values. Nevertheless, the representation is exactly equivalent to quantum mechanics. A number of states violating Bell inequalities are sampled, demonstrating that these quantum paradoxes can be treated with probabilistic methods. We treat quantum dynamics by simulating the time evolution of the Bell state formed via parametric down-conversion, and discuss multi-mode generalizations.
\end{abstract}

\section{INTRODUCTION}

The importance and complexity of quantum dynamics has been emphasized by many physicists, including Dirac and Feynman [1, 2]. We live in a dynamical, multi-mode universe described by quantum mechanics, yet the equations involved quickly become too complex to solve. Quantum simulations provide a means of carrying out such dynamical calculations. In principle one may do this using either computational methods [3 8 , or through a physical system that imitates the required properties 2, 9 13. However, while physical imitations can be useful, they are often unavailable with the required parameter values. Universal quantum computers are another possibility, and realizations of up to 6 qubits now exist [14, but this is too small for many problems.

Probabilistic quantum phase-space methods, which are potentially scalable, are often the only practical route towards quantum simulation of large systems [15. Hence, this approach is of great utility when simulating a multimode quantum system. This is because number state methods are unable to handle exponential complexity. Methods such as linearization fail when there are nonlinear effects. However, these issues do not greatly increase the complexity of probabilistic phase-space equations. The number of variables required increases linearly, not exponentially, with the number of spatial modes.

Such methods have been widely used to treat quantum dynamical problems in quantum optics and atom optics. They have been used to model propagation of radiation fields in superfluorescence [3], reproducing the observed delay statistics. They have been applied to dynamical propagation of quantum solitons, where the observed entanglement and squeezing was predicted to very high accuracy, including non-Markovian thermal reservoirs [4, 16]. Simulations of the quantum dynamics of critical points in parametric down-conversion have been carried out 17. Recently, very large three dimensional systems of colliding Bose-Einstein condensates have been treated [5, 18, 19]. These are first-principles, multi-mode, quantum dynamical calculations of substantial complexity, which show the potential of phase-space techniques.

An important question is whether these approaches can treat highly non-classical states. We are especially interested in cases that violate Bell inequalities. Here we investigate this issue, by demonstrating that Bell states have a probabilistic mapping which can be computationally sampled. Our motivation is to illustrate how these techniques work, in a situation where the quantum behavior is readily understood. We give a careful analysis of the different types of Bell inequalities, their loopholes when present, and the techniques required to simulate them, both for static and dynamic simulations. In this latter case we focus on how the positive P-distribution can be used to perform simulations of the time-evolution of the violation of Bell type inequalities. The models used can be readily scaled to larger multi-mode treatments.

We treat bipartite states using the Clauser-Horne 20] $(\mathrm{CH})$ version of the Bell inequalities [21, the ClauserHorne-Shimony-Holt (CHSH) inequalities [22, and the multi-particle generalizations of the $\mathrm{CH}$ inequalities 23 , 24 which are used in photonic experiments and we called CHD inequality. Our main focus here is on dynamical quantum simulations of these different Bell violations. This requires an analysis of the different types of measurement strategy used in these experiments. A summary of the static results for the CHD Bell inequality is published elsewhere [25]. In Section [V] we explain in depth both the computational strategy that allows probabilistic sampling of the positive-P distribution and the methods used to obtain evaluate the correlations for the static results of the CHD Bell inequality, as well as the other inequalities. We also compare the relative sampling errors obtained in the dynamic and static cases.

In the dynamical simulations, which illustrate these issues in specific cases relevant to experiment, we simulate the simplest model of the loophole-free violation of Bell type inequalities in parametric down conversion experiments [26 29], using the positive P-representation to simulate different types of Bell violation. For the CHSH Bell inequality we also take account of the post-selection process that is often used in experiment, which excludes null events. Surprisingly, these time-dependent quantum simulations of Bell inequality violations have lower sampling errors than static cases. We also describe how to extend our simple model to complex multi-mode systems. 
The phase-space distributions used in this paper are positive [30, 31]. They exist for all quantum states, and their statistical moments correspond to correlations of the type observed in Bell violations. We focus here on the usual photonic Bell state experiments which have been experimentally studied, with an emphasis on recent parametric down-conversion experiments [26, 28, 32, 33, in order to give a simple model for dynamical simulations. Large-scale Bell violations of multipartite systems have been treated elsewhere 34.

In order to understand this approach, we emphasize some important points. While stochastic, this technique is very different to conventional path-integral MonteCarlo methods. Path-integrals can give probabilistic behavior for ground states or finite temperature steady states, but they are not positive for real time dynamical evolution. Since quantum systems have no objective reality until measured, phase-space quantum simulation methods do not need to give dynamical evolution in terms of classical paths. We therefore use a more general definition of simulation. The probabilistic sampling that we employ gives quantum physical moments \langle\rangle$_{q m}$ as equal to probabilistic averages \langle\rangle$_{s t}$ over variables that may not be eigenvalues of observables. Thus, the mean value for the Pauli spin $\hat{\sigma}_{x}$ is given as $\left\langle\hat{\sigma}_{x}\right\rangle_{q m}=\left\langle\sigma_{x}\right\rangle_{s t}$ where the stochastic variable $\sigma_{x}$ can assume values beyond the eigenvalue spectrum, $1 / 2$ and $-1 / 2$. Such properties are closely associated with the quantum notion of "weak measurements" and "weak values" 35. From the perspective of quantum mechanics, this difference is not important, as long as one can predict experimentally measurable correlations and operator averages.

The paper is organized as follows. First, in Section II we review and analyze several different Bell inequalities, in order to examine which inequalities are most suitable for simulating a loophole-free Bell test based on parametric down conversion - which does not generate a simple Bell state. In Section III we discuss the positive P-representation, while Section IV treats static quantum simulation of a Bell state. In Section $\nabla$ we demonstrate the quantum dynamical simulations of violations of the $\mathrm{CH}$ and $\mathrm{CHSH}$ inequalities in parametric down conversion. Finally, Section VI summarizes our conclusions.

\section{BELL INEQUALITIES}

The assumption of a local hidden variable (LHV) theory (which assumes local realism) leads to a constraint a Bell inequality - on the observable correlations of a physical system [21]. These inequalities can be violated by quantum mechanics. In this paper our goal is to provide a probabilistic quantum simulation of these violations. There are many different Bell inequalities. Here, we describe only the Bell inequalities to be considered in this paper. We focus on three cases: The ClauserHorne-Shimony-Holt (CHSH) inequality, the ClauserHorne $(\mathrm{CH})$ inequality and the $\mathrm{CH}$ Bell inequality using moments extended to $N$ photon pairs, which we will call the $N$-photon CHD inequality.

\section{A. CHSH Inequality}

The CHSH Bell inequality was formulated to account for Bell's original proposal, where two particles are emitted from a common source and the measurement performed on each of the particles gives a binary outcome. It allows rigorous tests of LHV theories in realistic experimental scenarios where losses can be important.

In the case of two particles emitted from a common source, measurements by spatially separated observers (usually called Alice and Bob) are modeled in the LHV theory by taking random samples of a common set of parameters (the hidden variables) symbolized by $\lambda$. Here, $P(\lambda)$ denotes the probability distribution for the hidden variables $\lambda$, which can be discrete or continuous. Measured values are then functions of some local detector/ analyzer settings $a$ and $b$ at each location, and the hidden parameters $\lambda$. The value observed by Alice with detector settings $a$ is $A(a, \lambda)$, and similarly $B(b, \lambda)$ is defined for Bob's value with detector settings $b$. For binary outcomes, $A, B= \pm 1$.

We now introduce the specific notation of $A(\theta, \lambda)$ and $B(\phi, \lambda)$ for Alice and Bob with a variable analyzer setting $\theta$ and $\phi$ respectively. In most experiments so far, $\theta$ and $\phi$ correspond to polarizer angles [32, 33, 36, 37. Here, the measurement event includes the selection of the measurement settings $\theta, \phi$ at each site, done after the emission of the particles. The measurement events are assumed to be space-like separated. In a local hidden variable theory the correlations are thus obtained from a probabilistic calculation of the form:

$$
E(A, B)=\int A(\theta, \lambda) B(\phi, \lambda) P(\lambda) d \lambda .
$$

Clauser, Horne, Shimony and Holt obtained a version of Bell's inequality known as the CHSH Bell inequality [22, 38], which gives classical limits to the expected correlation for the above experiment conducted by Alice and Bob, and is given by $E[A, B]-E\left[A, B^{\prime}\right]+E\left[A^{\prime}, B\right]+$ $E\left[A^{\prime}, B^{\prime}\right] \leq 2$, where $A, A^{\prime}$ and $B, B^{\prime}$ are two sets of measurements made by Alice and Bob. We rewrite this as

$$
E(\theta, \phi)-E\left(\theta, \phi^{\prime}\right)+E\left(\theta^{\prime}, \phi\right)+E\left(\theta^{\prime}, \phi^{\prime}\right) \leq 2 .
$$

Here $E(\theta, \phi)$ is the correlation, $\theta$ and $\theta^{\prime}$ are measurements at location $\mathcal{A}$ with two different analyzer settings, while $\phi$ and $\phi^{\prime}$ are the corresponding measurements at location $\mathcal{B}$. From Tsirelson's theorem [39], it is known that $2 \sqrt{2}$ is the true upper bound for the left side of this inequality within quantum mechanics, and that the operators giving this maximal value can always be isomorphically mapped to the Pauli spin matrices. In the simulation graphs, we 
will plot a normalized ratio and its LHV bound as:

$$
\begin{aligned}
S_{\mathrm{CHSH}} & =\frac{1}{2}\left[E(\theta, \phi)-E\left(\theta, \phi^{\prime}\right)+E\left(\theta^{\prime}, \phi\right)+E\left(\theta^{\prime}, \phi^{\prime}\right)\right] \\
& \leq 1 .
\end{aligned}
$$

To violate the LHV bound quantum mechanically, one well-known route proposed by Bell is for Alice and Bob to make Pauli spin measurements on the spatially separated yet entangled particles created by the spin- $1 / 2$ singlet state [21]. Alternatively, any measurements with binary outcomes that have equivalent correlations in quantum mechanics will suffice, for example, polarization entangled photon pairs [37, 40.

Such "Bell" states are described by the wave-function:

$$
|\psi\rangle=\frac{1}{\sqrt{2}}\left(|1\rangle_{a}|-1\rangle_{b}-|-1\rangle_{a}|1\rangle_{b}\right)
$$

where here $| \pm 1\rangle_{a / b}$ represents the eigenstate of Pauli spin operator $S_{Z}$ for the system $a / b$. The spin measurements performed are as follows:

$$
\begin{aligned}
\hat{A} & =S_{z} \otimes I, \\
\hat{A}^{\prime} & =S_{x} \otimes I, \\
\hat{B} & =-\frac{1}{\sqrt{2}} I \otimes\left(S_{z}+S_{x}\right), \\
\hat{B}^{\prime} & =\frac{1}{\sqrt{2}} I \otimes\left(S_{z}-S_{x}\right) .
\end{aligned}
$$

A calculation within quantum mechanics shows that, for the Bell state, the Bell inequality is predicted to be violated:

$$
\langle\hat{A} \hat{B}\rangle-\left\langle\hat{A} \hat{B}^{\prime}\right\rangle+\left\langle\hat{A}^{\prime} \hat{B}\right\rangle+\left\langle\hat{A}^{\prime} \hat{B}^{\prime}\right\rangle=2 \sqrt{2}>2 .
$$

\section{B. Clauser-Horne Bell inequality}

The Clauser-Horne $(\mathrm{CH})$ inequality was developed to test LHV theories in situations relating to photons and polarizers where one of the outcomes may not be detectable. The $\mathrm{CH}$ inequality has proved useful for tests of LHV theories where the effect of detection inefficiencies is significant. We discuss this inequality, because it will also prove useful in establishing rigorous Bell tests of LHV theories where we use parametric down conversion (PDC) as the photon pair source [26] 28, 32, 33].

This test is designed to detect nonclassical behavior in the prototypical photonic Bell state, which is:

$$
\left|1_{B}\right\rangle=\frac{1}{\sqrt{2}}\left(\hat{a}_{1}^{\dagger} \hat{b}_{1}^{\dagger}+\hat{a}_{2}^{\dagger} \hat{b}_{2}^{\dagger}\right)|0\rangle .
$$

Here the relative phase of the two terms is irrelevant it just changes the polarizer settings - and we choose a positive sign for convenience. In the measurements, we consider two modes of orthogonal polarization incident on the polarizer at $\mathcal{A}$, and a second pair of orthogonal modes incident on the polarizer at $\mathcal{B}$. The modes at $\mathcal{A}$ and $\mathcal{B}$ are denoted by boson operators $\hat{a}_{1}\left(\hat{a}_{2}\right)$ and $\hat{b}_{1}$ $\left(\hat{b}_{2}\right)$ respectively. The two polarizers are independently rotated to settings $\theta$ and $\phi$. At each polarizer there are two possible output fields: the transmitted and reflected modes.

If a single photon is incident on one of the polarizers, there are two outcomes which we label "up" and "down" depending on whether the photon is detected in the transmitted or reflected mode respectively. The transmitted and reflected modes at polarizer $\mathcal{A}$ are defined by rotated operators

$$
\begin{aligned}
& \hat{c}_{+}=\hat{a}_{1} \cos \theta+\hat{a}_{2} \sin \theta \\
& \hat{c}_{-}=-\hat{a}_{1} \sin \theta+\hat{a}_{2} \cos \theta .
\end{aligned}
$$

Similarly, at $\mathcal{B}$ the two outputs are defined by boson operators:

$$
\begin{aligned}
& \hat{d}_{+}=\hat{b}_{1} \cos \phi+\hat{b}_{2} \sin \phi \\
& \hat{d}_{-}=-\hat{b}_{1} \sin \phi+\hat{b}_{2} \cos \phi .
\end{aligned}
$$

An experimentalist can measure at each location whether a single photon passes into the "up" or "down" mode at the polarizer. The outcome of measurement is assigned the Pauli spin value +1 if "up", and -1 if "down". In this way, one can establish the joint probability $P_{++}^{A B}(\theta, \phi)$ for detecting one photon at $\mathcal{A}$ "up" (i.e., in mode $c_{+}$) with setting $\theta$, and one photon "up" (i.e., in mode $d_{+}$) at $\mathcal{B}$ with setting $\phi$. It is also possible to measure the marginal probability $P_{+}^{A}(\theta)$ for detecting "up" at $\mathcal{A}$ with setting $\theta$. The marginal $P_{+}^{B}(\phi)$ is defined similarly.

We note that there can be a third outcome (apart from "up" and "down") at each detector. This is the null event where no photon is detected. This outcome is usually given the value $A, B=0$. The null outcome can occur either because of non-ideal detection efficiencies, or because of the nature of the quantum state describing the incident photon. For example, the state could be a vacuum state. For the parametric down conversion (PDC) process, there will be a nonzero probability for detecting zero and multiple photons at each "up" and "down" location.

Now we introduce the Clauser-Horne $(\mathrm{CH})$ Bell inequality which is satisfied for all LHV theories [20, 36]:

$$
\begin{aligned}
& P_{++}^{A B}(\theta, \phi)-P_{++}^{A B}\left(\theta, \phi^{\prime}\right)+P_{++}^{A B}\left(\theta^{\prime}, \phi\right)+P_{++}^{A B}\left(\theta^{\prime}, \phi^{\prime}\right) \\
& \quad \leq P_{+}^{A}\left(\theta^{\prime}\right)+P_{+}^{B}(\phi) .
\end{aligned}
$$

This type of Bell inequality has been studied extensively in the literature [23, 24, 41, 45]. It is useful in establishing loophole-free violations of LHV theories where the probability $\eta$ for detecting a photon incident on a detector is reduced below unity [32, 33].

For convenience in comparing graphs, we will define a 
normalized Bell inequality and its LHV bound as:

$$
\begin{aligned}
S_{\mathrm{CH}} & =\frac{P_{++}^{A B}(\theta, \phi)-P_{++}^{A B}\left(\theta, \phi^{\prime}\right)+P_{++}^{A B}\left(\theta^{\prime}, \phi\right)+P_{++}^{A B}\left(\theta^{\prime}, \phi^{\prime}\right)}{P_{+}^{A}\left(\theta^{\prime}\right)+P_{+}^{B}(\phi)} \\
& \leq 1 .
\end{aligned}
$$

The $\mathrm{CH}$ inequality may be compared with the ClauserHorne-Shimony-Holt (CHSH) Bell inequality (2) which can be applied in this case:

$$
E(\theta, \phi)-E\left(\theta, \phi^{\prime}\right)+E\left(\theta^{\prime}, \phi\right)+E\left(\theta^{\prime}, \phi^{\prime}\right) \leq 2,
$$

(or the version of the CHSH inequality introduced by Garg and Mermin [46]). Here,

$$
E(\theta, \phi)=P_{++}^{A B}(\theta, \phi)+P_{--}^{A B}(\theta, \phi)-P_{-+}^{A B}(\theta, \phi)-P_{+-}^{A B}(\theta, \phi)
$$

is the expected value of the product of the Pauli spin outcomes at each detector.

Low efficiencies $\eta$ will make violation of the inequalities 10 and 12 impossible, since the marginal probabilities on the right side of the $\mathrm{CH}$ inequality scale as $\eta$, whereas the joint probabilities on the left side of both inequalities scale as $\eta^{2}$. One approach is to evaluate the inequalities over the sub-ensemble of jointly detected counts only, but this introduces assumptions that create the so-called "detection loophole" 47. Where detection efficiencies are fully taken into account, the $\mathrm{CH}$ inequality generally becomes favorable compared to the $\mathrm{CHSH}$ Bell inequality, because the ratio of left to right side is $\eta$ as compared to $\eta^{2}$.

Experimentally, it has proved difficult to obtain a direct, loophole free violation of these Bell inequalities, owing to detector inefficiencies [42, 44, 45]. With simulations, we can include efficiency factors or not, as we choose; in this paper we simulate efficient detection. Nonetheless, the above reasoning also motivates us to use the $\mathrm{CH}$ Bell inequality for loophole-free tests where correlated joint null events are significant, as in the PDC process. We will explain this in a later section.

\section{Bell inequalities with intensity moments}

For the experimental scenario described in the previous subsection, one can reformulate the $\mathrm{CHSH}$ and $\mathrm{CH}$ Bell inequalities in terms of intensity correlations [23, 26]. This is useful for our calculation of the inequalities using probabilistic simulations, as it gives a reduced sampling error.

Consider the CHSH inequality. Since only one or zero photons is ever detected at each detector, the spin product $E(\theta, \phi)$ can be written as

$$
E(\theta, \phi)=\left\langle\left(\hat{c}_{+}^{\dagger} \hat{c}_{+}-\hat{c}_{-}^{\dagger} \hat{c}_{-}\right)\left(\hat{d}_{+}^{\dagger} \hat{d}_{+}-\hat{d}_{-}^{\dagger} \hat{d}_{-}\right)\right\rangle .
$$

Similarly for the $\mathrm{CH}$ inequality, the probability $P_{++}^{A B}(\theta, \phi)$ becomes

$$
P_{++}^{A B}(\theta, \phi)=\left\langle\hat{c}_{+}^{\dagger} \hat{c}_{+} \hat{d}_{+}^{\dagger} \hat{d}_{+}\right\rangle
$$

and the marginal probability $P_{+}^{A}(\theta)$ is

$$
P_{+}^{A}(\theta)=\left\langle\hat{c}_{+}^{\dagger} \hat{c}_{+}\right\rangle
$$

In this case, where the number of photons incident on each detector is always less than or equal to 1 , the Bell inequality expressed in terms of the intensity moments can be derived rigorously (since always $\mathrm{A}, B \leq 1$, as explained in Section II.A) without any extra assumptions that might introduce loopholes.

\section{D. $N$ - photon CHD Bell inequalities}

Following Drummond [23] and Reid [24, we also consider Bell inequalities defined where one has more than one photon incident on each detector, for the experimental scenario described above. Consider the case where $N$ photons are incident at each analyzer/detector. For example, we might consider a source that emits correlated photon pairs in the state

$$
\left|N_{B}\right\rangle=\frac{\left(\hat{a}_{1}^{\dagger} \hat{b}_{1}^{\dagger}+\hat{a}_{2}^{\dagger} \hat{b}_{2}^{\dagger}\right)^{N}|0\rangle}{N !(N+1)^{1 / 2}} .
$$

This state is a $2 N$ photon generalization of the Bell singlet state, which can be realized by a PDC [24, 48. One way to treat this is to define higher spin outcomes 49 . However, it is also useful to extend the $\mathrm{CH}$ approach, and to redefine the "+" event to be that where all $N$ photons are transmitted through the polarizer and, therefore, are detected in mode + .

We define the higher order intensity correlations

$$
G^{I J}(\theta, \phi, N)=\left\langle N\left|\left(\hat{c}_{+}^{\dagger}\right)^{I} \hat{c}_{+}^{I}\left(\hat{d}_{+}^{\dagger}\right)^{J} \hat{d}_{+}^{J}\right| N\right\rangle .
$$

These are proportional to the probability of observing $I$ photons of polarization + at detector $A$ and $J$ photons of polarization + at detector $B$ (exactly for $I=J=N$ but only as a first approximation otherwise). Then, we see that

$$
P_{++}^{A B}(\theta, \phi)=\kappa G^{N N}(\theta, \phi, N),
$$

where $\kappa$ is a proportionality constant. The marginal $P_{+}^{A}(\phi)$ can be defined by the correlation

$$
G^{I J}(\theta, \infty, N)=\left\langle N\left|\left(\hat{c}_{+}^{\dagger}\right)^{I} \hat{c}_{+}^{I}:\left(\hat{b}_{+}^{\dagger} \hat{b}_{+}+\hat{b}_{-}^{\dagger} \hat{b}_{-}\right)^{J}:\right| N\right\rangle .
$$

The " $\infty$ " case and the corresponding correlation $G^{I J}(\theta, \infty, N)$ stand for the same measurement as for $G^{I J}(\theta, \phi, N)$, but with no polarizer at the second detector. Since $N$ photons are incident at each detector, the marginal is given by

$$
P_{+}^{A}(\theta)=\kappa G^{N N}(\theta, \infty, N),
$$

and similarly for $P_{+}^{B}(\phi)$. This of course is useful, because for the $\mathrm{CH}$ inequality, the $\kappa$ constant will cancel, 
which means we do not have to worry about calculating its value and also that its smallness will not detract from a violation. We emphasize as above, that for the case where there are strictly $N$ photons incident at each location, the marginal can be calculated with the summation over all possible outcomes at the first detector (the $\infty$ term). Where there are also null events (zero detections) at one detector but not the other - as with inefficient detectors - the use of the joint correlation to describe the marginal will lead to loopholes.

For the state $\left|N_{B}\right\rangle$, which is a generalization of the Bell singlet state, the correlation function will depend only on the magnitude of the angle difference $\theta-\phi$. To simplify, we let this difference be called $\varphi$. Also, for the states of interest, the marginals are independent of $\theta$ or $\phi$, and there is symmetry so that $P_{+}^{A}(\theta)=P_{+}^{B}(\phi)$. On looking at the $\mathrm{CH}$ inequality, we then see that with the usual angle choice whereby $\theta, \phi, \theta^{\prime} \phi^{\prime}$ increase sequentially by $\varphi$ [21], the inequality has the form [23]:

$$
S_{\mathrm{CHD}}(\varphi) \equiv S_{N}^{N}(\varphi)=\frac{3 g_{N}^{N}(\varphi)-g_{N}^{N}(3 \varphi)}{2} \leq 0
$$

where

$$
g_{N}^{J}(\varphi)=G^{J J}(0, \varphi, N) / G^{J J}(0, \infty, N) .
$$

This expression generalizes the usual $\mathrm{CH}$ and Bell expressions to a multi-particle form. We will call this Bell inequality the $N$-photon CHD Bell inequality. The quantum mechanical prediction for $g_{N}^{J}$ for the state $|N\rangle$ has an especially simple form in case of $J=N: g_{N}^{N}(\varphi)=$ $\cos ^{2 N} \varphi$, which we use to test our simulations.

\section{E. Bell inequalities for parametric down conversion}

One objective of this paper is to simulate the dynamical generation of the quantum state that violates a Bell inequality. In recent experiments, violation of Bell inequalities has been achieved using parametric down conversion (PDC). In this section, we therefore introduce a simple dynamical model for this process, and consider which Bell inequalities can be used to test LHV theories for PDC generated states.

Usually, in the experiments, the statistics are evaluated only on a sub-ensemble of the measurement events. The sub-ensemble includes only those events whereby a single photon is detected at each of the spatially separated polarizers. In that case, it as though the Bell state was incident on the two polarizers, and traditional approaches (mentioned above) to testing Bell inequalities can be applied to test local realism. For the case of the $N$-photon Bell inequalities, one can similarly restrict to a $2 N$-particle sub-ensemble. Such projected measurements are typical of quantum optical tests of Bell inequalities. This projection can be arranged in principle as a form of state preparation ("heralding") 38]. Also, it can be arranged rigorously in the derivation of the
CH-Bell inequality by the suitable definition of the "+" measurement events [24], a process we will describe below and will refer to as "event selection". Both these forms of projection enable in principle a loophole free test. However, more commonly, the projection is created by "post-selection", after the detection of the photons. The post-selection procedure admits a loophole, but has been necessary because of the null events created by the inefficiency of detectors.

In this paper, we model the state generation by solving a Hamiltonian that describes the PDC process [24, 26], and consider testing Bell inequalities both with and without projection. Here, we must address the null events that are created by the PDC process. We show that it is possible to treat these events rigorously without introducing loopholes, provided one uses the right Bell inequality.

The experiments that test Bell's inequalities typically use either an atomic cascade or parametric downconversion, where the effective Hamiltonian has the form:

$$
\hat{H}=i \hbar \kappa E\left(\hat{a}_{1}^{\dagger} \hat{b}_{1}^{\dagger}-\hat{a}_{1} \hat{b}_{1}\right)+i \hbar \kappa E\left(\hat{a}_{2}^{\dagger} \hat{b}_{2}^{\dagger}-\hat{a}_{2} \hat{b}_{2}\right) \text {. }
$$

where $\kappa$ denotes the strength of the parametric interaction, and $E$ the strength of the pump field, and we will take $\hbar=1$. Here $\hat{a}_{1}^{\dagger}$ creates a photon in site $A$ with polarization 1 ("+"), $\hat{a}_{2}^{\dagger}$ creates a photon in site $\mathcal{A}$ with polarization 2 ("-"); similarly $\hat{b}_{1}^{\dagger}\left(\hat{b}_{2}^{\dagger}\right)$ creates a photon in site $\mathcal{B}$ with polarization $1(2)$. We suppose that $\hat{a}_{1,2}^{\dagger}$ $\left(\hat{b}_{1,2}^{\dagger}\right)$ creates a photon in the spatial modes detected at site $\mathcal{A}(\mathcal{B})$ with one of orthogonal polarizations 1 (2). We denote these modes by $A 1, A 2, B 1$ and $B 2$.

This model has just four relevant modes. By comparison, real experiments are typically inhomogeneous and multi-mode in character [27-29, 48], since experimentalists usually employ traveling wave packets with pulsed pump inputs to create the required spatial separation of detection events. Such issues are readily treated with phase-space methods, and full quantum field simulations have been treated elsewhere 50 52. However, our purpose is not to exactly model an experiment, since the details are different in every case. Instead, we wish to use this simple model to understand the fundamental issues of probabilistic sampling of quantum systems that violate a Bell inequality. A summary of the required changes to generalize the simulations is given in Section $\mathrm{VC}$.

The four-mode Hamiltonian generates a correlated squeezed state, with the generic form for $\kappa E t \ll 1$ of:

$$
\begin{aligned}
|\psi\rangle= & \exp (-i \hat{H} t)|0\rangle \\
= & |0\rangle+\kappa E t\left(|1\rangle_{A 1}|0\rangle_{A 2}|1\rangle_{B 1}|0\rangle_{B 2}\right. \\
& \left.+|0\rangle_{A 1}|1\rangle_{A 2}|0\rangle_{B 1}|1\rangle_{B 2}\right)+O(\kappa E t)^{2} .
\end{aligned}
$$

This does not generate just the Bell state $\left|1_{B}\right\rangle$. Instead, for $\kappa E t \ll 1$ it generates a linear superposition of the Bell state and a correlated vacuum state 
$|0\rangle \equiv|0\rangle_{A 1}|0\rangle_{A 2}|0\rangle_{B 1}|0\rangle_{B 2}$. Then, the generated quantum state has the form:

$$
|\psi\rangle \approx|0\rangle+c\left|1_{B}\right\rangle
$$

where $c=\sqrt{2} \kappa E t$.

Earlier, we considered the Clauser-Horne $(\mathrm{CH})$ Belltype inequality

$$
\begin{aligned}
& P_{++}^{A B}(\theta, \phi)-P_{++}^{A B}\left(\theta, \phi^{\prime}\right)+P_{++}^{A B}\left(\theta^{\prime}, \phi\right)+P_{++}^{A B}\left(\theta^{\prime}, \phi^{\prime}\right) \\
& \quad \leq P_{+}^{A}\left(\theta^{\prime}\right)+P_{+}^{B}(\phi) .
\end{aligned}
$$

Here, we can define the "+" event at each detector to be where $N$ photons are detected at the "+" polarized mode and a total of $N$ photons are detected in total at the "+" and "-" modes [24]. The CH Bell inequality is effective for loophole-free tests in the presence of correlated joint null events, which are significant in the PDC process due to the presence of the correlated vacuum state $|0\rangle$, the leading term for low $\kappa E t$. These null events will lead to a reduction in the absolute value of the joint probabilities (such as $P_{++}^{A B}(\theta, \phi)$ ), which substantially reduces the violation of the CHSH-type inequalities, unless heralding or some other strategy can be utilized. For the case of the ideal parametric amplifier, the joint null events are correlated. As a result, because the $\mathrm{CH}$ inequality is normalized by the marginals on the right-side, these vacuum events will have no impact on the violation of the $\mathrm{CH}$ inequality (27). Also, for PDC, the event of a total of $N$ particles being detected at one polarizer is correlated with a total of $N$ particles detected at the other polarizer. Therefore, this strategy is useful for projecting out the $N$-particle Bell state. We call this strategy "event selection" and note it is useful in providing loophole-free tests for the $N$-photon CHD Bell inequalities (using PDC).

In the following sections we explain how to probabilistically simulate the dynamics of the generation of Bell violations for PDC experiments, by using the simple model (24).

\section{POSITIVE P-REPRESENTATION}

There are a number of different positive phase-space representations. For bosonic systems, the most general class known extends the $s$-ordered representations of Cahill and Glauber [53] to include the set of all Gaussian operator bases [54, 55], defined over nonclassical phasespace coordinates.

\section{A. Definition and existence properties}

The most well-known of these nonclassical phase-space methods is the positive P-representation [30, which generalizes the Glauber-Sudarshan P-representation [56, 57] to all quantum states.
For $M$ bosonic modes, this is a non-unique expansion of an arbitrary density matrix $\hat{\rho}$ in coherent state projectors:

$$
\hat{\rho}=\int P\left(\vec{\alpha}, \vec{\alpha}^{+}\right) \hat{\Lambda}\left(\vec{\alpha}, \vec{\alpha}^{+}\right) d^{2 M} \vec{\alpha} d^{2 M} \vec{\alpha}^{+},
$$

where $\hat{\Lambda}$ is a coherent state projection operator, defined as:

$$
\hat{\Lambda}\left(\vec{\alpha}, \vec{\alpha}^{+}\right)=\frac{|\vec{\alpha}\rangle\left\langle\left(\vec{\alpha}^{+}\right)^{*}\right|}{\left\langle\left(\vec{\alpha}^{+}\right)^{*} \mid \vec{\alpha}\right\rangle}
$$

Here $|\vec{\alpha}\rangle=\left|\alpha_{1} \ldots \alpha_{M}\right\rangle$ is a multi-mode coherent state of a bosonic field, which is an eigenstate of the corresponding operators $\left(\hat{a}_{1}, \ldots \hat{a}_{M}\right)$. The probability function $P\left(\vec{\alpha}, \vec{\alpha}^{+}\right)$is defined on an enlarged, nonclassical phase-space, which allows positive probabilities for all quantum states.

This representation maps bosonic quantum states into $4 M$ real coordinates:

$$
\begin{aligned}
\vec{\alpha} & =\vec{p}+i \vec{x} \\
\vec{\alpha}^{+} & =\vec{p}^{+}+i \vec{x}^{+},
\end{aligned}
$$

which is double the dimension of the corresponding classical phase-space. This method leads to exact probabilistic mappings between quantum mechanics and a classical-like phase-space description, even for low occupation numbers. It is often advantageous to perform a variable change to sum and difference variables:

$$
\begin{aligned}
& \vec{\nu}=\left(\vec{\alpha}-\left(\vec{\alpha}^{+}\right)^{*}\right) / 2, \\
& \vec{\mu}=\left(\vec{\alpha}+\left(\vec{\alpha}^{+}\right)^{*}\right) / 2 .
\end{aligned}
$$

A general probabilistic construction using these variables, which is non-unique but always exists, is [30]:

$$
P\left(\vec{\alpha}, \vec{\alpha}^{+}\right)=\frac{\langle\vec{\mu}|\widehat{\rho}| \vec{\mu}\rangle}{(2 \pi)^{2 M}} e^{-|\vec{\nu}|^{2}} .
$$

We will use this distribution for the static sampling calculations. However, our dynamical sampling calculations do not employ this form, but rather use a dynamically generated distribution, obtained from solving coupled stochastic equations.

In all cases, with the positive P-distribution, the expectation of any normally ordered observable $\hat{O} \equiv$ $O\left(\hat{a}_{1}^{\dagger}, \hat{a}_{1}, \ldots\right)$ is:

$$
\langle\hat{O}\rangle=\int O\left(\alpha_{1}^{+}, \alpha_{1}, \ldots\right) P\left(\vec{\alpha}, \vec{\alpha}^{+}\right) d^{2 M} \vec{\alpha} d^{2 M} \vec{\alpha}^{+} .
$$

For Bell state measurements, the effects of a polarizer are simply obtained on taking linear combinations of mode amplitudes, just as in classical theory or with quantum operators [26]. If we represent the input operators $\left(\hat{a}_{1}, \hat{a}_{2}, \hat{b}_{1}, \hat{b}_{2}\right)$ by complex variables $\left(\alpha_{1}, \alpha_{2}, \beta_{1}, \beta_{2}\right)$, 
the transmitted and reflected modes at polarizer $A$ are defined by rotated complex phase-space variables:

$$
\begin{aligned}
& \gamma_{+}=\alpha_{1} \cos \theta+\alpha_{2} \sin \theta \\
& \gamma_{-}=-\alpha_{1} \sin \theta+\alpha_{2} \cos \theta,
\end{aligned}
$$

for the "up" and "down" modes respectively. Similarly, at $B$ the outputs are defined by boson operators:

$$
\begin{aligned}
& \delta_{+}=\beta_{1} \cos \theta+\beta_{2} \sin \theta \\
& \delta_{-}=-\beta_{1} \sin \theta+\beta_{2} \cos \theta,
\end{aligned}
$$

The corresponding hermitian conjugate terms are represented by replacing $\alpha, \beta, \gamma, \delta$ by independent complex variables $\alpha^{+}, \beta^{+}, \gamma^{+}, \delta^{+}$. The advantage is that this can represent entangled states: a positive $\mathrm{P}$-function always exists for any density matrix. In particular, it exists for the photonic Bell state.

Since this is always probabilistic, there is a great similarity between the hidden variable theory (1) of Bell, and the positive-P formula (33) for quantum correlations, from setting $\lambda=\left(\vec{\alpha}, \vec{\alpha}^{+}\right)$. However, while the hidden variable theory obeys Bell's theorem and hence cannot be equivalent to quantum theory, the positive-P theory is fully equivalent to quantum mechanics, and therefore can violate Bell's inequalities.
The reason for the difference is due to the different quantities calculated in the correlations [26]. The fundamental observables in Bell's case, of the form $X(\lambda)$, are defined as being equal to actual observed real numbers, that is, $(0,1, \ldots)$ for photon counts. The corresponding observables in the positive-P case, of form $n\left(\vec{\alpha}, \vec{\alpha}^{+}\right)$, are complex numbers whose mean values and correlations correspond to observable means and correlations. Given these unrestricted numbers, the proof of the Bell inequality is no longer applicable.

This difference allows the positive P-distribution to be exactly equivalent to quantum mechanics, even though it appears in other respects just like a probabilistic hidden variable theory. As a result, this approach is well-suited to carrying out probabilistic quantum simulations. This property of having quasi-observable parameters different to eigenvalues, is also shared by weak quantum measurement strategies 35 .

\section{B. Cooperative Bell state distribution}

The four-mode state (17) has the corresponding positive P-distribution [23]:

$$
P\left(\vec{\alpha}, \vec{\alpha}^{+}\right)=\left\{\frac{\left|\left(\alpha_{1}^{+}+\alpha_{1}^{*}\right)\left(\beta_{1}^{+}+\beta_{1}^{*}\right)+\left(\alpha_{2}^{+}+\alpha_{2}^{*}\right)\left(\beta_{2}^{+}+\beta_{2}^{*}\right)\right|^{2 N}}{(2 \pi)^{8}(N+1)(N !)^{2} 2^{4 N}}\right\} \exp \left(-\frac{|\vec{\alpha}|^{2}+\left|\vec{\alpha}^{+}\right|^{2}}{2}\right) .
$$

For the positive-P function in the form of 32 , we perform the variable change given in Eqn. (31), which has a Jacobian $2^{2 M}$. For this four-mode distribution of interest, we additionally introduce four complex vector functions that describe the phase-space variables corresponding to measurements at $\mathrm{A}$ and $\mathrm{B}$ respectively, giving a total of 16 real dimensions. These are:

$$
\begin{aligned}
\vec{A} & =\left[\mu_{A 1}, \mu_{A 2}\right], \quad \vec{B}=\left[\mu_{B 1}, \mu_{B 2}\right], \\
\delta \vec{A} & =\left[\nu_{A 1}, \nu_{A 2}\right], \quad \delta \vec{B}=\left[\nu_{B 1}, \nu_{B 2}\right] .
\end{aligned}
$$

Then the positive P-distribution (36) can be written in the form of:

$$
P(\vec{A}, \vec{B}, \delta \vec{A}, \delta \vec{B})=P(\vec{A}, \vec{B}) G(\delta \vec{A}) G(\delta \vec{B}) .
$$

Here we have introduced an auxiliary distribution of:

$$
P(\vec{A}, \vec{B})=\left(\frac{|\vec{A} \cdot \vec{B}|^{2 N}}{\pi^{4}(N+1)(N !)^{2}}\right) e^{-|\vec{A}|^{2}-|\vec{B}|^{2}}
$$

together with normal distributions:

$$
G(\delta \vec{A})=\frac{1}{\pi^{2}} e^{-|\delta \vec{A}|^{2}} .
$$

\section{SAMPLING METHOD AND STATIC BELL VIOLATIONS}

In order to use probabilistic methods for the static distributions given in the previous section, it is necessary to have a computational algorithm that generates probabilistic samples.

We can sample $\delta \vec{A}, \delta \vec{B}$ as four dimensional Gaussian variates in real space, with a real variance of $\sigma^{2}=1 / 2$ in each real coordinate. Here we note that:

$$
\int e^{-|\vec{A}|^{2}} d^{2} \vec{A}=\left[\pi \int e^{-R} d R\right]^{2}=\pi^{2} .
$$

Hence, the 8 real difference coordinates can all be exactly sampled without rejection.

\section{A. Von Neumann Rejection Algorithm}

To sample $P(\vec{A}, \vec{B})$ over the 8 remaining real variables, we choose the well-known von Neumann rejection algorithm, which is an easily implemented technique. The algorithm used here relies on sampling with a distribution proportional to a positive, normalizable function $\tilde{F}(\vec{A}, \vec{B})$ 
that is always larger than the target distribution. Once sampled, the numbers generated are randomly accepted or rejected in proportion to $P / \tilde{F}$, to obtain samples with the required distribution. Since it is clear that:

$$
|\vec{A}, \vec{B}| \leq|\vec{A}||\vec{B}|
$$

we can choose the computational function according to:

$$
P(\vec{A}, \vec{B}) \leq \tilde{F}(\vec{A}, \vec{B})=F(\vec{A}) F(\vec{B}),
$$

where $F(\vec{A})$ has the following structure:

$$
F(\vec{A})=\frac{|\vec{A}|^{2 N}}{\pi^{2} \sqrt{N+1} N !} \exp \left(-|\vec{A}|^{2}\right) .
$$

Hence, we can use the rejection method described above. We note that it might be feasible to use hyperspherical coordinates and sample this without rejection, but as the algorithm described here works well, we did not attempt this refinement.

The function $F(\vec{A})$ has to be normalized to establish the acceptance/rejection ratio. In order to do that, we notice that:

$$
\begin{aligned}
\int|\vec{A}|^{2 N} \exp \left(-|\vec{A}|^{2}\right) d^{k} \vec{A} & =S_{k-1}(1) \int_{0}^{\infty} r^{2 N+k-1} e^{-r^{2}} d r \\
& =\frac{1}{2} \Gamma(N+k / 2) S_{k-1}(1), \quad(45)
\end{aligned}
$$

where $k$ is the number of components in $\vec{A}$. In this case $\vec{A}$ contains two complex numbers, so $k=4$, and $S_{k-1}(r)$ is the surface area of a $k$-dimensional ball:

$$
S_{k-1}(r)=\frac{2 \pi^{k / 2} r^{k-1}}{\Gamma(k / 2)} .
$$

Therefore, the normalization gives:

$$
\begin{aligned}
\mathcal{N} & =\int F(\vec{A}) d^{4} \vec{A} \\
& =\frac{\Gamma(N+2)}{2 \pi^{2} \sqrt{N+1} N !} \times \frac{2 \pi^{2}}{\Gamma(2)} . \\
& =\sqrt{N+1}
\end{aligned}
$$

and $F(\vec{A})=\mathcal{N} \tilde{P}(\vec{A})$, where $\tilde{P}$ is a probability distribution:

$$
\tilde{P}(\vec{A})=\frac{|\vec{A}|^{2 N}}{\pi^{2}(N+1) !} \exp \left(-|\vec{A}|^{2}\right)
$$

which is a combination of a lambda-distribution of the vector length and a uniform distribution of its direction and therefore can be sampled exactly.

It can be represented as a product of two independent distributions [58]:

$$
\tilde{P}(r, \vec{n})=S_{k-1}(r) g\left(r^{2}\right) U(\vec{n})=R(r) U(\vec{n}),
$$

where $r=|\vec{A}|, \vec{n}$ is a unit vector on a $k$-dimensional sphere, and $U=1 / S_{k-1}(1)$ is a uniform distribution of vector directions (or, in other words, a uniform distribution on the surface of a $k$-dimensional ball). The distribution of directions can be sampled by sampling a vector of $k$ normally distributed random numbers and normalizing it to 1 [59, 60]. In order to sample the distribution of lengths, we have to do another change of variable: $r^{2} \rightarrow x$, so that:

$$
\begin{aligned}
R(x) & =\frac{1}{2 \sqrt{x}} S_{k-1}(\sqrt{x}) g(x) \\
& =\frac{1}{2 \sqrt{x}} \frac{2 \pi^{k / 2} x^{(k-1) / 2}}{\Gamma(k / 2)} \frac{x^{N}}{\pi^{2}(N+1) !} \exp (-x) \\
& =\frac{x^{N+1}}{\Gamma(N+2)} \exp (-x) .
\end{aligned}
$$

The result is exactly the gamma distribution with a shape parameter $N+2$.

\section{B. Probabilistic violation of a Bell inequality}

In Figs. 1 and 2 we give computational results that show the probabilistic violation of the bipartite $N$-photon CHD Bell inequality of Eqn. (22), for polarized photons of the state (17). Here we use $N=1$ and $N=2$ photons pairs respectively. In both figures the dotted line corresponds to the quantum mechanical prediction. Also plotted is the corresponding dynamical calculation, which is explained in the next section.

We have plotted the violation of $S_{C H D}$ defined in $(22)$, where the correlations $G^{I J}$ were calculated using averages of the corresponding phase space variable moments. In order to evaluate the $N$-photon CHD Bell inequality, the relevant correlations are given by:

$$
\begin{aligned}
G^{I J}(\theta, \phi, N) & =\left\langle\left|\gamma_{+}\right|^{2 I}\left|\delta_{+}\right|^{2 J}\right\rangle_{P} \\
G^{I J}(\theta, \infty, N) & =\left\langle\left|\gamma_{+}\right|^{2 I}\left(\left|\beta_{+}\right|^{2}+\left|\beta_{-}\right|^{2}\right)\right\rangle_{P} .
\end{aligned}
$$

These results indicate a clear violation of the Bell inequality in both the standard two-particle case $(N=1)$ and the four-particle case $(N=2)$. This has also been observed experimentally [48]. The computational results demonstrate a complete agreement with quantum predictions up to the sampling error. This shows that these Bell violations can certainly be simulated probabilistically.

The simulated Bell violations for this inequality are shown in Figs. 1 for the $N=1$ case, and in Fig. 2 for the $N=2$ case. This demonstrates a clear violation of a Bell inequality using a probabilistic simulation, in both cases. The graphs include results from a static simulation just of the Bell state, and also from a dynamical simulation of a typical experiment using parametric down-conversion, which will be explained next. We used $2^{18}$ trajectories for dynamic simulations, $2^{18}$ samples for the static sampling with $N=1$, and $2^{24}$ samples for the static sampling with $N=2$ (to accommodate for the quickly growing 


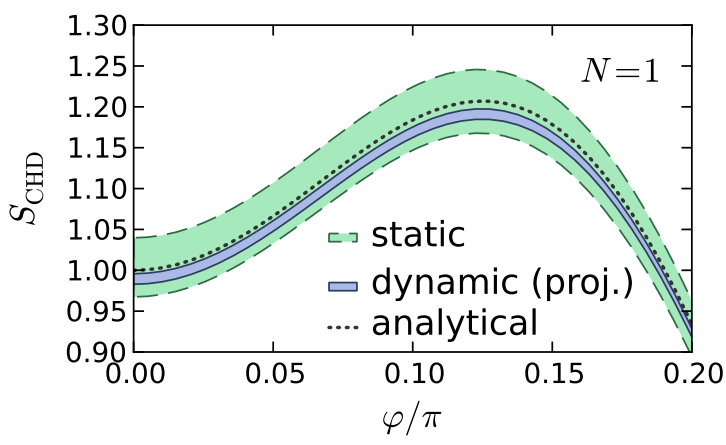

Figure 1. (Color online) Simulated moment-based CHD Bell violation $S_{\mathrm{CHD}}(\varphi)$ as a function of the relative polarizer angle $\varphi$ for one photon pair using the positive P-distribution. Green dashed lines show the result of static sampling with $2^{18}$ samples. Blue solid lines show the results of the dynamic simulation with $2^{18}$ trajectories at dimensionless time $\tau=0.1$. For each of the sampled states, the filled region represents the range of the estimated error around the mean of $S_{\mathrm{CHD}}(\varphi)$. The exact quantum mechanical prediction of this value is represented by the grey dotted line.

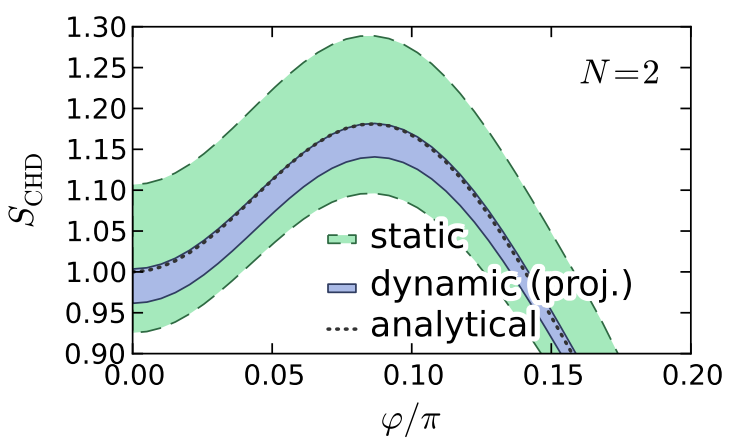

Figure 2. (Color online) Simulated moment-based Bell violation $S_{\mathrm{CHD}}(\varphi)$ as a function of the relative polarizer angle $\varphi$ for two photon pairs using the positive P-distribution. Green dashed lines show the result of static sampling with $2^{24}$ samples. Blue solid lines show the results of a dynamic simulation with $2^{18}$ trajectories at dimensionless time $\tau=0.1$. For each of the sampled states, the filled region represents the range of the estimated error around the mean of $S_{\mathrm{CHD}}(\varphi)$. The exact quantum mechanical prediction of this value is represented by the grey dotted line.

sampling error in the static case). The sampling error could be reduced if we used more samples.

We could instead have investigated the violations of other Bell inequalities that use state probabilities rather than moments. All these inequalities are operationally equivalent for the states used here, as explained in Section II.C. However, state-projection calculations give larger sampling errors than moments when using the standard 'canonical' positive-P distribution of Eqn. (36). This non-unique form is easily computed, but it is more suitable for calculating moments rather than probabilities.
There are other expressions for the positive-P distribution, as well as alternative representations like the general Gaussian representations [54 which are better for sampling probabilities 61, but are outside the scope of this article. The $\mathrm{CH}$ and $\mathrm{CHSH}$ state projection Bell inequalities will be treated in the next section, which deals with quantum dynamics.

\section{DYNAMICAL SIMULATIONS}

In this section we explain the model used to perform dynamical simulations for the $\mathrm{N}$-photon CHD-Bell inequality, together with the $\mathrm{CH}$ and $\mathrm{CHSH}$ inequalities. In this latter case we also include the post-selection/ heralding process. This dynamical approach, as well as being more physically realistic, has lower sampling errors than the static calculations. The reason for this is that the dynamical equations generate a more compact phase-space distribution, which is readily calculated using stochastic methods. This improved sampling efficiency more than compensates for the need to calculate time evolution, which is rather straightforward.

\section{A. Dynamical simulations for the $N$-photon CHD inequality}

In order to illustrate quantum dynamical simulations of violations of Bell type inequalities using the positive Prepresentation, we will consider the process of parametric down conversion described earlier, which is modeled by the effective Hamiltonian of Eqn. (24). The positive Prepresentation provides a mapping that transforms the time evolution of a density matrix into a set of phase space stochastic equations.

For the Hamiltonian of Eqn. (24) we obtain the following set of stochastic equations [17, 26]:

$$
\begin{aligned}
d \alpha_{1} & =\kappa E \beta_{1}^{+} d t+\sqrt{\kappa E} d W_{1} \\
d \beta_{1} & =\kappa E \alpha_{1}^{+} d t+\sqrt{\kappa E} d W_{1}^{*} \\
d \alpha_{2} & =\kappa E \beta_{2}^{+} d t+\sqrt{\kappa E} d W_{2} \\
d \beta_{2} & =\kappa E \alpha_{2}^{+} d t+\sqrt{\kappa E} d W_{2}^{*} \\
d \alpha_{1}^{+} & =\kappa E \beta_{1} d t+\sqrt{\kappa E} d W_{1}^{+} \\
d \beta_{1}^{+} & =\kappa E \alpha_{1} d t+\sqrt{\kappa E}\left(d W_{1}^{+}\right)^{*} \\
d \alpha_{2^{+}} & =\kappa E \beta_{2} d t+\sqrt{\kappa E} d W_{2}^{+} \\
d \beta_{2}^{+} & =\kappa E \alpha_{2} d t+\sqrt{\kappa E}\left(d W_{2}^{+}\right)^{*},
\end{aligned}
$$

where the only non-vanishing correlations are

$$
\left\langle d W_{i} d W_{j}^{*}\right\rangle=\left\langle d W_{i}^{+}\left(d W_{j}^{+}\right)^{*}\right\rangle=d t \delta_{i j} .
$$

This set of Stratonovich stochastic equations of (52) can be solved numerically in order to find the complex variables $\alpha_{i}(t), \beta_{i}(t)$ as a function of time. The rotated 
complex phase-space variables $\gamma_{+}(t)$ and $\delta_{+}(t)$ are defined in Eqns. (34) and (35) respectively. We use these complex variables to evaluate the intensity correlations for one photon pair, described in Eqn. (18), as well as the time evolution of the Bell type inequality of Eqn. (22).

The expressions for the intensity correlations that we use are given, as in the static case, by Eqn. (51). We recall that the dimensionless parametric interaction squeeze parameter $r$ is proportional to $\kappa E t$, and accordingly, we graph our results against a dimensionless time $\tau=\kappa E t$.

In Figs. 1 and 2 we show the results of dynamical simulations for $N=1$ and $N=2$ photons pairs respectively using the moment-based CHD Bell inequality. These figures are plotted at $\tau \leq 0.25$, which we found was a suitable time that minimizes the production of unwanted higher spin multiple pairs.

Here we find that the sampling error in the dynamical case is smaller that the static case. This is because the static distribution we used has a simple existence theorem, but is non-unique, and usually does not give the minimum variance possible. For all dynamical simulations we used the central difference numerical algorithm 62, with coupling $\kappa E=1$ and time step $d t=2 \times 10^{-4}$, which is sufficient to make discretization error negligible.

At very short times we observe a large sampling error. There is a very clear physical reason for this. For these times the distribution is dominated by the photonic vacuum state, giving a large sampling error due to the fluctuations in the projection operator for the Bell states. At times larger than about $\tau=0.1$, the onset of multiple pair production occurs, which starts to reduce the Bell violation, as we no longer have an ideal state.

\section{B. Dynamical simulations for the PDC process}

In order to obtain the time evolution of the violation of the Clauser-Horne and CHSH Bell type inequalities using the positive P-representation, we will now derive the appropriate operator mappings. As described above, we use the positive P-representation in order to obtain the complex variables $\alpha_{i}(t), \beta_{i}(t)$ as a function of time and also the complex variables $\gamma_{i}(t), \delta_{i}(t)$, which are defined through the equations (34) and (35) respectively.

\section{CH inequality}

The next step is the evaluation of each of the probabilities of the $\mathrm{CH}$ inequality, Eqn. (27), as well as all the probabilities of the CHSH inequality of Eqn. (12). Let us consider one of these probabilities, for instance the probability of detecting one photon in the up position at the polarizer with location A and one photon in the "up" position at location $\mathrm{B}, P_{++}^{A B}(\theta, \phi)$, which is evaluated as

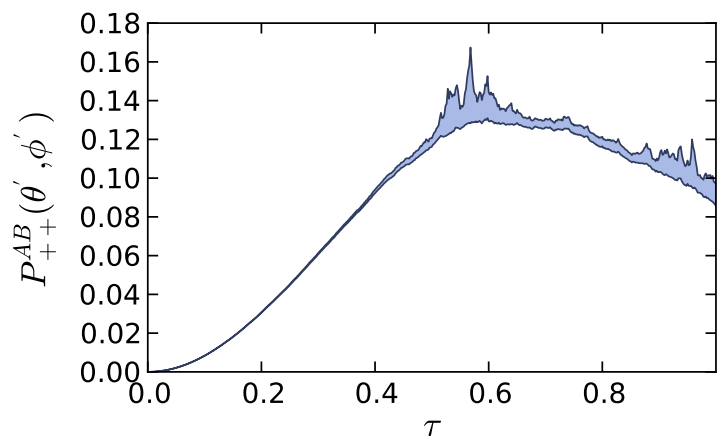

Figure 3. (Color online) Evolution of a single probability $P_{++}^{A B}\left(\theta^{\prime}, \phi^{\prime}\right)$ demonstrating that the sampling error increases after $t=0.5$.

follows:

$$
\begin{aligned}
P_{++}^{A B}(\theta, \phi) & =\operatorname{Tr}(\hat{\rho}|1100\rangle\langle 1100|) \\
& =\int P\left(\vec{\gamma}, \vec{\gamma}^{+}\right) \frac{\langle 1100 \mid \vec{\gamma}\rangle\left\langle\left(\vec{\gamma}^{+}\right)^{*} \mid 1100\right\rangle}{\left\langle\left(\vec{\gamma}^{+}\right)^{*} \mid \vec{\gamma}\right\rangle} \\
& =\int P\left(\vec{\gamma}, \vec{\gamma}^{+}\right) e^{-\vec{\gamma}^{+} \cdot \vec{\gamma}} \gamma_{1} \gamma_{1}^{+} \delta_{1} \delta_{1}^{+} d \vec{\gamma} d \vec{\gamma}^{+}(54)
\end{aligned}
$$

Here $\vec{\gamma}=\left(\gamma_{1}, \gamma_{2}, \delta_{1}, \delta_{2}\right)$ and $\vec{\gamma}^{+}=\left(\gamma_{1}^{+}, \gamma_{2}^{+}, \delta_{1}^{+}, \delta_{2}^{+}\right)$. The other probabilities are evaluated similarly. The marginal probabilities $P_{+}^{A}(\theta)$ and $P_{+}^{B}(\phi)$ are evaluated as follows:

$$
\begin{aligned}
P_{+}^{A}(\theta) & =\int P\left(\vec{\gamma}, \vec{\gamma}^{+}\right) e^{-\left(\gamma_{1}^{+} \gamma_{1}+\gamma_{2}^{+} \gamma_{2}\right)} \gamma_{1}^{+} \gamma_{1} d \vec{\gamma} d \vec{\gamma}^{+}, \\
P_{+}^{B}(\phi) & =\int P\left(\vec{\gamma}, \vec{\gamma}^{+}\right) e^{-\left(\delta_{1}^{+} \delta_{1}+\delta_{2}^{+} \delta_{2}\right)} \delta_{1}^{+} \delta_{1} d \vec{\gamma} d \vec{\gamma}^{+} .
\end{aligned}
$$

To test the Clauser-Horne inequality, we evaluate the predictions for $P_{++}^{A B}(\theta, \phi)$ and $P_{+}^{A}(\theta)$, for the state created by the Hamiltonian (24). Here, we have defined the "+" event to be the detection of a single photon at the up position, which means that the number of photons at the down position does not need to be detected. This is the original formulation of the $\mathrm{CH}$ inequality.

In our simulations we noticed that after $\tau=0.5$ the sampling error increased, as indicated by additional fluctuations after this time in Fig. 3, which shows the evolution of a single probability $P_{++}^{A B}\left(\theta^{\prime}, \phi^{\prime}\right)$. The sampling error increase at long times is due to a larger proportion of four and six particle states, and a correspondingly increased distribution radius in phase-space. However, this is not optimal for Bell violations, for which the short-time behavior is more physically important, as experimentally these higher photon numbers are not utilized. Accordingly, we take our samples at relatively short times with $\tau<0.25$ in this paper, which is the most physically relevant time-scale. Experimentally this corresponds to using a relatively short pump pulse or short interaction distance, since this controls the evolution time.

For the purpose of calculation, we can choose our basis $a_{+}$to correspond to the mode axis $c_{+}$defined by $\theta$ 
of polarizer $A$. This amounts to putting $\theta=0$ in the calculation. We note that the Hamiltonian $a_{+}^{\dagger} b_{+}^{\dagger}+a_{-}^{\dagger} b_{-}^{\dagger}$ is invariant under this type of rotation.

We can gain further insight into the solutions by writing the Hamiltonian as $H=H_{1}+H_{2}$, where $H_{1(2)}=$ $\kappa E \hat{a}_{1(2)} \hat{b}_{1(2)}+H c$. The evolution of the PDC is given by

$$
\begin{aligned}
|\psi\rangle & =e^{-i H t / \hbar}|0\rangle \\
& =e^{-i H_{1} t / \hbar} e^{-i H_{2} t / \hbar}|0\rangle \\
& =\left(\sum_{n=0} c_{n}|n\rangle_{a_{1}}|n\rangle_{b_{1}}\right)\left(\sum_{n=0} c_{n}|n\rangle_{a_{2}}|n\rangle_{b_{2}}\right),
\end{aligned}
$$

where we have taken the initial state to be the multimode vacuum state $|0\rangle$, which is the product of the vacuum states of each of the four modes. Since $e^{-i H_{+} t / \hbar}|0\rangle$ is by definition a two-mode squeezed state, we have used in the last line the well-known result for the expansion of the two-mode squeezed state in terms of the Fock number state basis. Here, the Fock state for mode $a$ is denoted $|n\rangle_{a}$ and $c_{n}=\frac{x^{n}}{\left(1-x^{2}\right)^{1 / 2}}$ where $x=\tanh r$ [63]. For small $r$, we can expand (56) as explained in Section IIE

$$
|\psi\rangle=\tilde{c_{0}}|0\rangle+\tilde{c_{1}}|1\rangle+\ldots,
$$

where $|1\rangle$ is a Bell state and $\tilde{c}_{0}=c_{0}^{2}, \tilde{c_{1}}=\sqrt{2} c_{0} c_{1}$. To gain an understanding of the predictions for the Bell inequalities in this limit, we transform to the modes of the measured basis $c_{+}$and $d_{+}$:

$$
\begin{aligned}
|1\rangle= & \frac{1}{\sqrt{2}}\left\{\left(c_{+}^{\dagger} d_{+}^{\dagger}+c_{-}^{\dagger} d_{-}^{\dagger}\right) \cos \varphi\right. \\
& \left.+\left(-c_{+}^{\dagger} d_{-}^{\dagger}+c_{-}^{\dagger} d_{+}^{\dagger}\right) \sin \varphi\right\}|0\rangle,
\end{aligned}
$$

where $\varphi=\phi-\theta$. Then, we see that

$$
P_{++}^{A B}(\theta, \phi)=P_{--}^{A B}(\theta, \phi)=\left|\tilde{c}_{1}\right|^{2} \frac{1}{2} \cos ^{2}(\theta-\phi),
$$

and

$$
P_{+-}^{A B}(\theta, \phi)=P_{-+}^{A B}(\theta, \phi)=\left|\tilde{c}_{1}\right|^{2} \frac{1}{2} \sin ^{2}(\theta-\phi),
$$

and the marginals are

$$
P_{+}^{A}(\theta)=P_{+}^{B}(\phi)=P_{-}^{A}(\theta)=P_{-}^{B}(\phi)=\left|\tilde{c}_{1}\right|^{2} / 2 .
$$

We note that $\left|\tilde{c}_{1}\right|^{2}$ is the probability that the PDC process generates the correlated photon-pair state, whereby a single photon is incident on each detector. Choosing the usual case where the angles $\theta, \phi, \theta^{\prime} \phi^{\prime}$ increase sequentially by $\pi / 8$ [21], we see that for the $\mathrm{CH}$ inequality 27) the left side is $\left\{\left|\tilde{c}_{1}\right|^{2} / 2\right\}(3(\cos (\pi / 4) / 2+1 / 2)-$ $(\cos 3 \pi / 4+1) / 2)=\left|\tilde{c}_{1}\right|^{2}(\sqrt{2}+1) / 2$ whereas the right side is $\left|\tilde{c}_{1}\right|^{2}$. Thus, in the limit of small $r$, the ratio of the left to right side of the $\mathrm{CH}$ inequality is predicted to be $(\sqrt{2}+1) / 2$, which, being greater than 1 , violates the prediction of LHV theories.

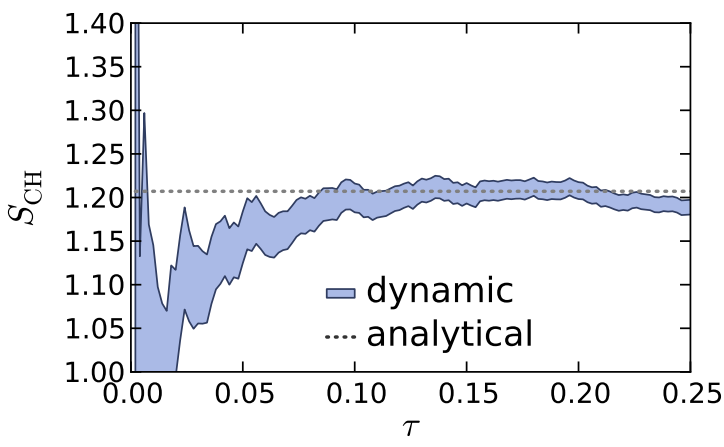

Figure 4. (Color online) Evolution of the violation of the Clauser-Horne Bell type inequality for the state generated by the parametric down conversion process 24): Plotted is the ratio $S_{\mathrm{CH}}$, defined in Eqn. 62 for the angle choices $\theta=0$, $\phi=\frac{\pi}{8}, \theta^{\prime}=\frac{\pi}{4}$ and $\phi^{\prime}=3 \frac{\pi}{8}$. Violation of the Bell inequality occurs when $S_{\mathrm{CH}}>1$. The filled region represents the range of the estimated error around the mean of $S_{\mathrm{CH}}$. The horizontal dotted line is the expected value with no high-order components. Here we consider $2^{18}$ samples.

This result is indeed evident from our full solution, plotted in Fig. 4, where we have defined:

$$
S_{\mathrm{CH}}=\frac{\tilde{P}(\theta, \phi)-\tilde{P}\left(\theta, \phi^{\prime}\right)+\tilde{P}\left(\theta^{\prime}, \phi\right)+\tilde{P}\left(\theta^{\prime}, \phi^{\prime}\right)}{P_{+}^{A}\left(\theta^{\prime}\right)+P_{+}^{B}(\phi)},
$$

where $\tilde{P} \equiv P_{++}^{A B}$. The violation of the $\mathrm{CH}$ inequality as shown when $S_{\mathrm{CH}}>1$ is a rigorous (loophole-free) test of LHV theories.

In Fig. 4 we show the time evolution simulations of the violations of the Clauser-Horne inequality using $2^{18}$ samples. Since we are considering a ratio in this case, the results are the same with or without the post-selection process. In fact post-selection - which could introduce loop-holes in principle for other Bell inequalities - simply has no effect on the measured data.

For higher $\tau$, correlated number states $|n\rangle|n\rangle$ where $n \geq 2$ will also contribute to the statistics. Since we have defined the "+" outcome to be that where a single photon is detected at the "up" position, the violation of the $\mathrm{CH}$ inequality (27) diminishes. This is because a + event at one detector can arise from either the single or multi-photon Fock states.

We note that the violation can be retained if one defines the + outcome differently, to be detection of one photon in the "up" position and one photon in the "down" position, as we explained above in Section (IIE) 24]. This latter definition amounts to the "event selection" method of projection of the Bell state, and would prove a loophole-free test, for larger times. Nonetheless, the probability of the actual measured "+" event becomes smaller in that case, and here we calculate the behavior of the original $\mathrm{CH}$ inequality (27) to show the dynamical evolution of the statistics.

We find that these quantum dynamical simulations give a clear violation of the Clauser-Horne inequality, 


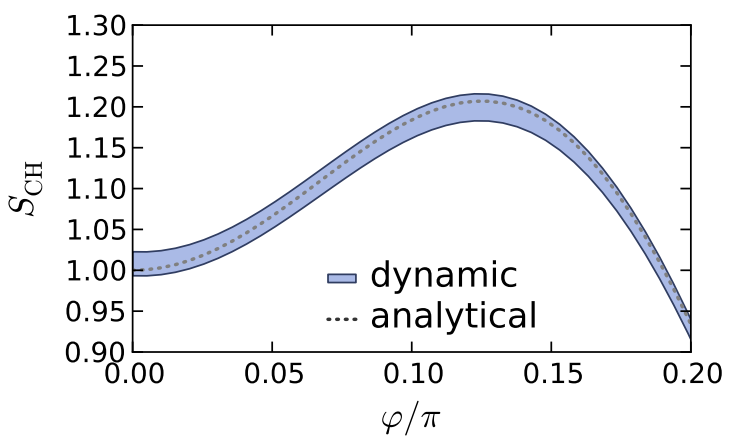

Figure 5. (Color online) Angular dependence of the simulated Clauser-Horne Bell type inequality for the state generated by the parametric down conversion process (24): Plotted is the ratio $S_{\mathrm{CH}}$, defined in Eqn. 62 as a function of the relative polarizer angle $\varphi=\phi-\theta=\phi^{\prime}-\theta^{\prime}=\theta^{\prime}-\phi$ at dimensionless time $\tau=0.1$. The filled region represents the range of the estimated error around the mean of $S_{\mathrm{CH}}$. Here we consider $2^{18}$ samples.

which occurs when when $S_{\mathrm{CH}}>1$. By choosing a particular time duration of $\tau=0.1$, we can examine the detailed predictions for angular correlations with respect to the relative polarizer angle $\varphi=\phi-\theta=\phi^{\prime}-\theta^{\prime}=\theta^{\prime}-\phi$. This is shown in Fig. 5 .

\section{Sampling the CHSH inequality with and without post-selection}

In our simulations, we also consider how to simulate the experimental post-selection/ heralding process, in which data is discarded in the case where no photon is detected (the null-event). In order to do this we consider a projection operator defined as:

$$
\hat{P}=\hat{1}-|0\rangle\langle 0| .
$$

In this case the density matrix will be of the form:

$$
\hat{\rho}^{\prime}=\frac{\hat{P}^{\dagger} \hat{\rho}(t) \hat{P}}{\operatorname{Tr}\left(\hat{P}^{\dagger} \hat{\rho}(t) \hat{P}\right)} .
$$

For the positive P-representation we will have a normalization given by:

$$
\operatorname{Tr}\left(\hat{P}^{\dagger} \frac{|\vec{\gamma}\rangle\left\langle\left(\vec{\gamma}^{+}\right)^{*}\right|}{\left\langle\left(\vec{\gamma}^{+}\right)^{*} \mid \vec{\gamma}\right\rangle} \hat{P}\right)=\left\langle 1-e^{-\vec{\gamma}^{+} \cdot \vec{\gamma}}\right\rangle .
$$

If we consider the post-selection process, the probabilities will have normalization factor of the form of Eqn. 65. For instance, if we consider the post-selection process for the probability $P_{++}^{A B}(\theta, \phi)$ that will be denoted as $P^{P}(|1100\rangle)$ we will obtain:

$$
P^{P}(|1100\rangle)=\frac{\left\langle\gamma_{1} \gamma_{1}^{+} \delta_{1} \delta_{1}^{+} e^{-\vec{\gamma}^{+} \cdot \vec{\gamma}}\right\rangle}{\left\langle 1-e^{-\vec{\gamma}^{+} \cdot \vec{\gamma}}\right\rangle} .
$$

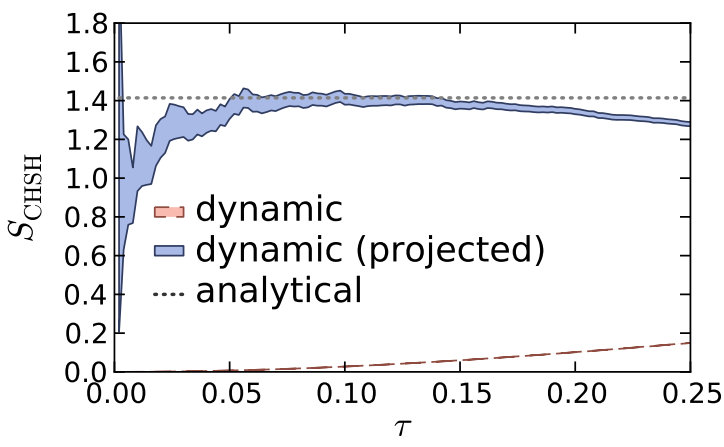

Figure 6. (Color online) Evolution of the violation of the CHSH Bell inequality for the state generated by the parametric down conversion process 24): Plotted is the ratio $S_{\mathrm{CHSH}}$, defined in Eqn. 67) for the angle choices $\theta=0, \phi=\frac{\pi}{8}, \theta^{\prime}=\frac{\pi}{4}$ and $\phi^{\prime}=3 \frac{\pi}{8}$. Violation of the Bell inequality occurs when $S_{\mathrm{CHSH}}>1$. The blue lines corresponds to the case with postselection/heralding where we consider the projector operator defined in Eqn. 63 and exclude the joint null events from the statistics, while the red lines corresponds to the simulation of the CHSH Bell-type inequality without post-selection. In each case, the filled region represents the range of the sampled error. The horizontal axis is the expected value at $t=0$.

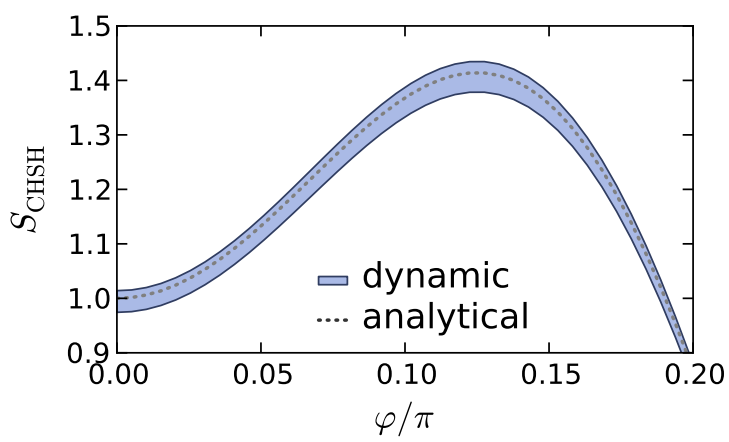

Figure 7. (Color online) Angular dependence of the simulated CHSH Bell inequality for the state generated by the parametric down conversion process (24): Plotted is the ratio $S_{\mathrm{CHSH}}$, defined in Eqn. (67) as a function of the relative polarizer angle $\varphi=\phi-\theta=\phi^{\prime}-\theta^{\prime}=\theta^{\prime}-\phi$ for dimensionless time $\tau=0.1$. In this case we consider the post-selection/ heralding process. The filled region represents the range of the sampled error.

Plotted in Fig. 6, are the predictions for the CHSH inequality, both with and without post-selection. As previously, we have defined

$$
S_{\mathrm{CHSH}}=\frac{E(\theta, \phi)-E\left(\theta, \phi^{\prime}\right)+E\left(\theta^{\prime}, \phi\right)+E\left(\theta^{\prime}, \phi^{\prime}\right)}{2},
$$

and we get violations when $S_{\mathrm{CHSH}}>1$.

In Fig. 7 we show the CHSH Bell type inequalities as a function of the relative polarizer angle $\varphi=\phi-\theta=$ $\phi^{\prime}-\theta^{\prime}=\theta^{\prime}-\phi$. The simulations were performed at $\tau=0.1$. In the figure we show the expected behavior for the CHSH inequality, including post-selection, as a function of the angle. Also plotted in these figures in the 
corresponding quantum mechanical prediction, showing excellent agreement.

\section{Extended simulations}

As an example of multi-mode problems which are of increasing interest in physics, Bell violation PDC experiments in the laboratory have much more complexity than the simple model we have used so far. Effects not present in our model include multi-mode spatial propagation, nonlinearity and loss. While it is the principle of probabilistic quantum simulation of Bell violations that is of interest here, scalability is also important. Therefore, we now show how our simulations can include such effects.

To treat these more realistic cases, we consider a waveguide based gedanken experiment. As our model, we suppose that a single pump field interacts with four down-converted waveguide modes, such that each pair of polarization modes is generated in a single waveguide. This is only one possible strategy to create a Bell violation. Laboratory experiments use a variety of approaches, with different details in each implementation.

While many methods are known experimentally, the extended model we treat here is chosen as it is the closest to the four-mode treatment given above, to allow a comparison. This waveguide proposal is actually more complex than waveguides currently used. The stochastic equations for this system have been obtained previously in simpler cases [52, 64, and we extend this earlier analysis using the same techniques.

The main conclusion one reaches is that the ordinary stochastic differential equations given in Eqn. (52) are replaced by a very similar set of partial stochastic differential equations for stochastic fields. We assume for simplicity that all group velocities are equal to $v$. The equations are [52, 64]:

$$
\begin{aligned}
& {\left[\frac{\partial}{\partial z}+\frac{i k^{\prime \prime}}{2} \frac{\partial^{2}}{\partial t_{v}^{2}}\right] \Phi_{i}^{a}=-\gamma \Phi_{i}^{a}+\kappa^{*} \Psi \Phi_{i}^{b+}+\sqrt{\kappa^{*} \Psi} \zeta_{i}} \\
& {\left[\frac{\partial}{\partial z}+\frac{i k^{\prime \prime}}{2} \frac{\partial^{2}}{\partial t_{v}^{2}}\right] \Phi_{i}^{b}=-\gamma \Phi_{i}^{b}+\kappa^{*} \Psi \Phi_{i}^{a+}+\sqrt{\kappa^{*} \Psi} \zeta_{i}^{*}} \\
& {\left[\frac{\partial}{\partial z}+\frac{i k_{p}^{\prime \prime}}{2} \frac{\partial^{2}}{\partial t_{v}^{2}}\right] \Psi=-\gamma_{p} \Psi-\kappa \sum_{i} \Phi_{i}^{a} \Phi_{i}^{b}}
\end{aligned}
$$

Here $\Psi$ is the stochastic pump field, while $\Phi_{i}^{a}$ and $\Phi_{i}^{b}$ are the down-converted fields for $i=1,2$. There are five equations for these fields, and five more independent equations for the corresponding fields $\Psi^{+}, \Phi_{i}^{a+}$ and $\Phi_{i}^{b+}$. All fields are flux amplitudes defined so that $\left\langle\Psi \Psi^{+}\right\rangle$is the photon flux, with field units of $s^{-1 / 2}$. This normalization is the most useful for the treatment of photon propagation.

The coordinate $z$ is the distance along the waveguide, $t_{v}=t-z / v$ is a moving frame time coordinate, $k^{\prime \prime}=d^{2} k / d \omega^{2}$ gives the group velocity dispersion, while $\gamma, \gamma_{p}$ are the amplitude loss rates. The noise terms $\zeta_{i}$ are defined as previously, except that they are now deltacorrelated both in time and space, rather than just in time. These equations include nonlinearity, multi-mode dispersion and coupling to a dissipative reservoir describing losses.

Apart from these modifications, solving these equations is very similar to the original stochastic differential equations, with robust numerical algorithms being available 65. Waveguide experiments of this type are known to be an efficient method of generating correlated photons [66], providing a useful alternative to atomic cascade [37] or bulk crystal PDC [67] experiments.

We note that the mode indices $a, b$ describe mode polarizations, while $i$ is a spatial mode index. The polarizations need to be swapped with a polarizing beam-splitter in order to obtain the correlated Bell state outputs that are required. Here, beam-splitting is a unitary operation which is obtained through linear combinations of stochastic terms. These issues are described in the original theoretical proposals for PDC methods [26, 27].

\section{CONCLUSIONS}

In his work on quantum computers 2, Feynman treated an example of Bell states with correlations equivalent to the Bell violating measurements we study. He showed that a probabilistic simulation was not possible with simulations that sample the observed eigenvalues, since they would be equivalent to hidden variable theories. This raises the question of whether other types of probabilistic simulations can be carried out for these states.

Our main result is very simple. There is no barrier to simulating Bell violations probabilistically. The reason is that our phase-space simulations do not use the operator eigenvalues. Instead, they employ complex values whose averages and correlations are the same as the known quantum correlations that violate a Bell inequality. For this reason, such probabilistic quantum simulation methods are not hidden variable theories, but are instead like quantum weak measurements [35. Therefore, they are not restricted to classical predictions that satisfy Bell inequalities.

The simulations treated here were carried out in a number of ways, either from known static phase-space distributions for the Bell state, or dynamically. Timedependent, dynamical simulations in fact are simpler to implement than the static ones. We have investigated three different types of Bell inequality, and have successfully simulated them all, although the moment-based Bell inequalities are more well-suited to the representation chosen here than ones using state projections and quantum probabilities. The chief limitation of these methods is their sampling error, which depends on the precise measurement simulated. Another issue is the growth rate of sampling errors, which tend to increase with time in 
a way that is not unlike the growth of error in many classical dynamical systems.

Classical simulations of quantum systems commonly are thought to require an exponentially large memory. With phase-space methods this requirement disappears, since the phase-space dimension is simply proportional to mode number. Instead, one must analyze the scaling of sampling errors, which depends on the correlation order and the number of modes and samples. This is analyzed in more detail in related investigations into multipartite correlations 34, 68, which reached a size of 60 qubits and 60-th order moments. In these papers it was shown that exponentially large numbers of samples can be required for simulations of mesoscopic quantum superpositions, in cases where measured correlations have an order comparable to the mode or qubit number. Such issues can result in exponentially long simulation times. In practice, issues of inefficiency and noise limit the order of correlations that can be measured in the laboratory [69], hence this is not usually a problem when simulating real experiments.

If the measured correlations have a more realistic fixed order, as in the present work, the scaling is much more favorable. Phase-space methods have already been shown to provide accurate results even for extremely large systems of bosons [4, 5], with such finite order moments. A more serious limitation of the present method is the growth of sampling errors in time, which provides a time horizon for accurate quantum predictions. We also emphasize that the specific techniques used here are for bosonic, not fermionic systems.

In summary, the positive-P representation method was used because it is a complete, positive representation of any bosonic quantum state, with known procedures for obtaining dynamical quantum simulations. This is especially useful in the case of the photonic parametric down-conversion experiments widely utilized for Bell violations. The technique can easily be expanded to treat more complex multi-mode Hamiltonians. Other methods for phase-space mappings exist as well [54, and some of these methods may converge even more rapidly.

\section{ACKNOWLEDGMENTS}

We wish to acknowledge funding from the Australian Research Council Discovery Grants program.
[1] P. A. M. Dirac, P. Roy. Soc. A. 123, 714 (1929).

[2] R. P. Feynman, Int. J. Theor. Phys. 21, 467 (1982).

[3] F. Haake, H. King, G. Schröder, J. Haus, and R. J. Glauber, Phys. Rev. A 20, 2047 (1979).

[4] J. F. Corney et al., Phys. Rev. Lett. 97, 023606 (2006)

[5] P. P. Deuar and P. D. Drummond, Phys. Rev. Lett. 98, $120402(2007)$

[6] O. E. Alon, A. I. Streltsov, and L. S. Cederbaum, Phys. Rev. A 77, 033613 (2008).

[7] J. Gambetta et al., Phys. Rev. A 77, 012112 (2008)

[8] S. Trotzky et al., Nat. Phys. 8, 325 (2012).

[9] J. I. Cirac and P. Zoller, Science 301, 176 (2003)

[10] D. Jaksch and P. Zoller, Ann. Phys. 315, 52 (2005)

[11] I. Buluta and F. Nori, Science 326, 108 (2009).

[12] R. Islam et al., Nat. Commun. 2, 377 (2011).

[13] I. M. Georgescu, S. Ashhab, and F. Nori, Rev. Mod. Phys. 86, 153 (2014)

[14] B. P. Lanyon et al., Science 334, 57 (2011).

[15] B. Opanchuk, R. Polkinghorne, O. Fialko, J. Brand, and P. D. Drummond, Ann. Phys. 525, 866 (2013).

[16] P. D. Drummond, R. M. Shelby, S. R. Friberg, and Y. Yamamoto, Nature 365, 307 (1993).

[17] K. Dechoum, P. D. Drummond, S. Chaturvedi, and M. D. Reid, Phys. Rev. A 70, 53807 (2004)

[18] P. P. Deuar, J. Chwedeńczuk, M. Trippenbach, and P. Ziń, Phys. Rev. A 83, 063625 (2011).

[19] V. Krachmalnicoff et al., Phys. Rev. Lett. 104, 150402 (2010)

[20] J. F. Clauser and M. A. Horne, Phys. Rev. D 10, 526 (1974)

[21] J. S. Bell, Physics 1, 195 (1964).

[22] J. F. Clauser, M. A. Horne, A. Shimony, and R. A. Holt, Phys. Rev. Lett. 23, 880 (1969).
[23] P. D. Drummond, Phys. Rev. Lett. 50, 1407 (1983)

[24] M. D. Reid, W. J. Munro, and F. De Martini, Phys. Rev. A 66, 033801 (2002).

[25] P. D. Drummond, B. Opanchuk, L. E. C. Rosales-Zárate, and M. D. Reid, Phys. Scripta T160, 014009 (2014).

[26] M. D. Reid and D. F. Walls, Phys. Rev. A 34, 1260 (1986)

[27] Y. H. Shih and C. O. Alley, Phys. Rev. Lett. 61, 2921 (1988)

[28] Z. Y. Ou and L. Mandel, Phys. Rev. Lett. 61, 50 (1988)

[29] P. G. Kwiat et al., Phys. Rev. Lett. 75, 4337 (1995).

[30] P. D. Drummond and C. W. Gardiner, J. Phys. A: Math. Gen. 13, 2353 (1980).

[31] M. Hillery, R. F. O'Connell, M. O. Scully, and E. P. Wigner, Phys. Rep. 106, 121 (1984).

[32] M. Giustina et al., Nature 497, 227 (2013).

[33] B. G. Christensen et al., Phys. Rev. Lett. 111, 130406 (2013).

[34] B. Opanchuk, L. E. C. Rosales-Zárate, M. D. Reid, and P. D. Drummond, Phys. Lett. A 378, 946 (2014)

[35] Y. Aharonov, D. Z. Albert, and L. Vaidman, Phys. Rev. Lett. 60, 1351 (1988).

[36] J. F. Clauser and A. Shimony, Rep. Prog. Phys. 41, 1881 (1978).

[37] A. Aspect, P. Grangier, and G. Roger, Phys. Rev. Lett. 49, 91 (1982).

[38] B. D'Espagnat, ed., Foundations of Quantum Mechanics (Academic Press, New York, 1971).

[39] B. S. Cirel'son, Lett. Math. Phys. 4, 93 (1980).

[40] A. Aspect, J. Dalibard, and G. Roger, Phys. Rev. Lett. 49, 1804 (1982)

[41] P. Eberhard, Phys. Rev. A 47, R747 (1993)

[42] A. Cabello, Phys. Rev. Lett. 98, 220402 (2007). 
[43] N. Brunner, N. Gisin, V. Scarani, and C. Simon, Phys. Rev. Lett. 98, 220403 (2007).

[44] J.-A. Larsson, Phys. Rev. A 57, 3304 (1998).

[45] J.-A. Larsson and J. Semitecolos, Phys. Rev. A 63, $022117(2001)$

[46] A. Garg and N. D. Mermin, Phys. Rev. D 35, 3831 (1987)

[47] P. M. Pearle, Phys. Rev. D 2, 1418 (1970)

[48] J. Howell, A. Lamas-Linares, and D. Bouwmeester, Phys. Rev. Lett. 88, 030401 (2002).

[49] N. D. Mermin, Phys. Rev. D 22, 356 (1980)

[50] S. J. Carter, P. D. Drummond, M. D. Reid, and R. M. Shelby, Phys. Rev. Lett. 58, 1841 (1987)

[51] P. D. Drummond and S. J. Carter, J. Opt. Soc. Am. B 4, 1565 (1987)

[52] M. J. Werner, M. G. Raymer, M. Beck, and P. D. Drummond, Phys. Rev. A 52, 4202 (1995).

[53] K. E. Cahill and R. J. Glauber, Phys. Rev. 177, 1857 (1969)

[54] J. F. Corney and P. D. Drummond, Phys. Rev. A 68, $063822(2003)$

[55] J. F. Corney and P. D. Drummond, Phys. Rev. Lett. 93, 260401 (2004)

[56] R. J. Glauber, Phys. Rev. 131, 2766 (1963).
[57] E. Sudarshan, Phys. Rev. Lett. 10, 277 (1963)

[58] A. K. Gupta and D. Song, J. Stat. Plan. Infer. 60, 241 (1997)

[59] G. Marsaglia, Ann. Math. Statist. 43, 645 (1972)

[60] M. E. Muller, Commun. ACM 2, 19 (1959)

[61] L. E. C. Rosales-Zárate and P. D. Drummond, Phys. Rev. A 84, 042114 (2011)

[62] P. D. Drummond and I. K. Mortimer, J. Comput. Phys. 93, 144 (1991).

[63] M. Hillery and M. S. Zubairy, Phys. Rev. Lett. 96, 050503 (2006)

64] M. G. Raymer, P. D. Drummond, and S. J. Carter, Opt. Lett. 16, 1189 (1991)

65] M. J. Werner and P. D. Drummond, J. Comput. Phys. 132, 312 (1997)

[66] A. B. U'Ren, C. Silberhorn, K. Banaszek, and I. A. Walmsley, Phys. Rev. Lett. 93, 093601 (2004).

[67] A. Fedrizzi, T. Herbst, A. Poppe, T. Jennewein, and A. Zeilinger, Opt. Express 15, 15377 (2007)

[68] M. D. Reid, B. Opanchuk, L. Rosales-Zárate, and P. D. Drummond, (2014), arXiv:1406.2432.

[69] P. P. Rohde, K. R. Motes, P. A. Knott, and W. J. Munro, (2014), arXiv:1401.2199 